\title{
Electrical transport through carbon nanotube junctions created by mechanical manipulation
}

\author{
Henk W. Ch. Postma, Mark de Jonge, Zhen Yao, and Cees Dekker \\ Department of Applied Physics and DIMES, Delft University of Technology, Lorentzweg 1, 2628 CJ Delft, The Netherlands
}

(Received 22 June 2000)

\begin{abstract}
Using an atomic force microscope we have created nanotube junctions such as buckles and crossings within individual single-wall metallic carbon nanotubes connected to metallic electrodes. The electronic transport properties of these manipulated structures show that they form electronic tunnel junctions. The conductance shows power-law behavior as a function of bias voltage and temperature, which can be well modeled by a Luttinger liquid model for tunneling between two nanotube segments separated by the manipulated junction.
\end{abstract}

Molecular electronics has taken a large step forward since the discovery of carbon-nanotube metallic and semiconducting molecular wires. ${ }^{1}$ Various nanotube devices have been found to behave as conventional electronic components. For instance, individual semiconducting nanotubes function as field-effect transistors at room temperature, ${ }^{2}$ while metallic nanotubes are single-electron transistors at low temperature. ${ }^{3,4}$ More recently, it was found that intramolecular metal-semiconductor kink junctions can act as rectifying diodes at room temperature. ${ }^{5}$ Unlike conventional solid-state devices, however, nanotubes are molecules. Conformational changes can, therefore, be expected to strongly affect the electronic properties of nanotubes, opening up a route towards nanoscale electromechanical devices. Indeed, theoretical work has indicated that local deformations such as twists and buckles may induce strong barriers for electron transport. $^{6-8}$ While some transport experiments have been conducted on carbon nanotube junctions which occur naturally ${ }^{5,9,10}$ and on defects due to locally applied strain, ${ }^{11}$ a focused study with control over the geometry and configuration of the junction is lacking.

Here, we report electron transport measurements on molecular junctions that have been fabricated in a controlled manner from straight undeformed nanotubes by manipulation with an atomic force microscope (AFM). We have fabricated nanotube buckles and crossings and characterized their electron transport properties. We find that these mechanically manipulated structures act as tunnel junctions with a conductance that show power-law dependences on both bias voltage and temperature. For various sample layouts we obtain a wide range of power-law exponents, from 0.25 to 1.4 . We show that this variety can be understood within one consistent Luttinger model.

Single-wall carbon nanotubes were produced by the group of Smalley at Rice University. A small amount of this raw material is ultrasonically dispersed and spin coated on top of a $\mathrm{SiO}_{2} / \mathrm{Si}$-substrate containing a large array of predefined $\mathrm{Pt}$ electrodes. These electrodes are fabricated using a double layer polymethylmethacrylate/metacrylic acid resist, electron beam lithography, reactive ion etching, Pt evaporation, and lift-off. The resulting electrodes are embedded in the $\mathrm{SiO}_{2}$ substrate such that the height difference between the electrodes and substrate is less than $1 \mathrm{~nm}$. Nanoscale tunnel junctions are then created within individual carbon nanotubes by use of the AFM. Conductance measurements are performed using a standard ac-lockin technique.

Figure 1 presents two examples of nanojunctions that were fabricated with an AFM from individual metallic carbon nanotubes. In the fabrication procedure, the tip of the AFM is used to change the lateral position of a nanotube lying on top of metallic electrodes. First, a nanotube is identified by scanning the tip over the sample in tapping-mode AFM. Then, the tip is pressed onto the surface and moved along a predefined path across the nanotube. In this manner, the position and shape of nanotubes can be controlled with a high degree of accuracy. ${ }^{12}$ In Fig. 1(a) we show the initial configuration of a straight nanotube lying across four electrodes. In order to bend the tube between the middle two electrodes, the nanotube has been dragged across the surface in a direction perpendicular to its length. During this dragging action, the nanotube has slided along its length across the electrodes. The sharp bend that results from the AFM
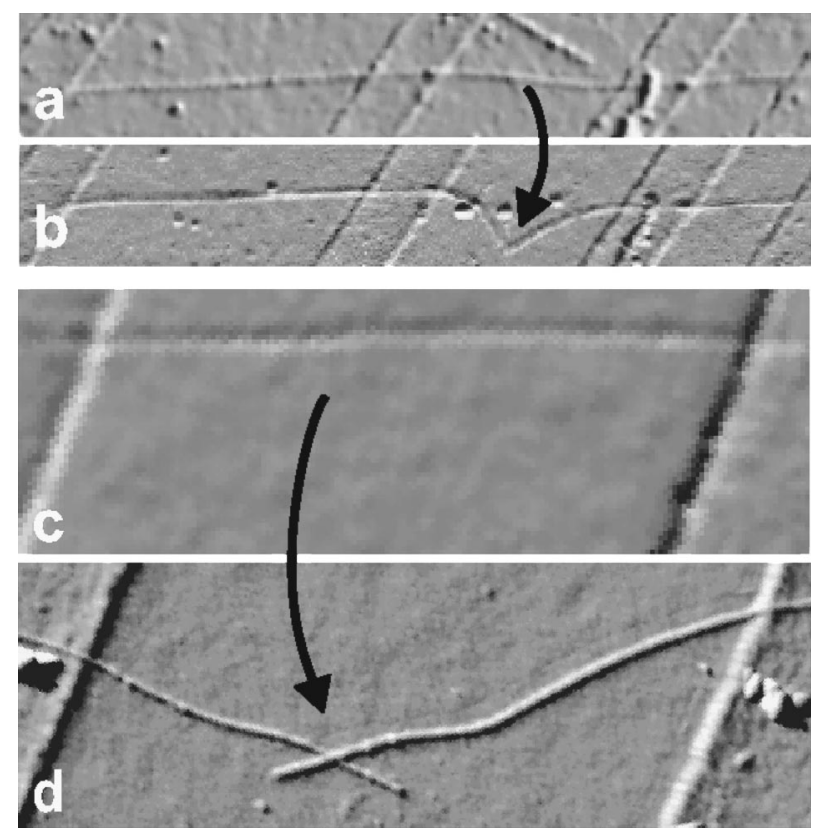

FIG. 1. Formation of carbon nanotube nanojunctions by AFM manipulation. Between the images in (a) and (b), an initially straight nanotube has been dragged to the bottom by the AFM tip, resulting in a sharp $105^{\circ}$ buckle. Image (c) and (d) show the manipulation of a nanotube crossing from an initially straight nanotube. The nanotube ends are extending 110 (left) and $130 \mathrm{~nm}$ (right) beyond the crossing point. The difference in apparent width of the nanotubes in these images is due to variation in the AFM tip radius which is different for different tips, and which moreover can change in the manipulation process. 


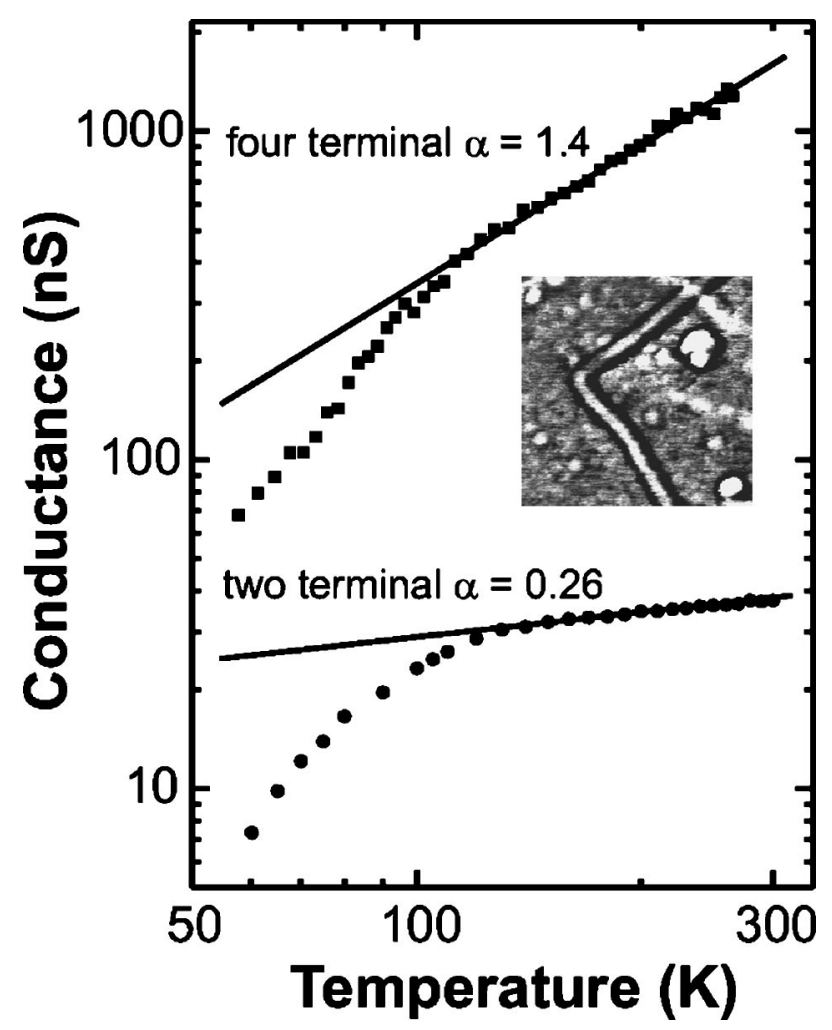

FIG. 2. Conductance of a nanotube buckle as a function of temperature in a four- and two-terminal measurement. The straight solid lines on this log-log plot indicate the power-law behavior $G$ $\propto T^{\alpha}$, with the exponent $\alpha$ as denoted. Below $120 \mathrm{~K}$, Coulomb blockade sets in which further suppresses the conductance at low temperatures. The inset shows a $300 \times 300 \mathrm{~nm}^{2}$ AFM phase image of the nanotube buckle. The four-terminal measurement reveals the intrinsic buckle conductance, whereas the two-terminal conductance is limited by the contact conductance.

manipulation has an angle of $105^{\circ}$ [see Fig. 1(b), and also inset to Fig. 2]. This is well above the critical value of about $60^{\circ}$ needed to form a so-called "buckle," 13 where a strongly bent nanotube releases strain by locally collapsing the cylindrical shell structure into a flattened tube structure. Accordingly, a small height increase is found at the bending point. Another example of a manipulated nanojunction is shown in Figs. 1(c) and 1(d). In this case, the dragging action of the AFM has broken the nanotube. Subsequently, the two broken ends of this nanotube have been pushed back together into a configuration where they cross each other. The resulting nanotube ends extend about $100 \mathrm{~nm}$ beyond the crossing point.

Multiterminal contacting of the nanotube allows one to separately measure the contact conductance (from two- and three-terminal measurements) and the intrinsic conductance of the manipulated tube (from a four-terminal measurement). The buckled nanotube sample in Fig. 1(b) has contacts with a low contact conductance, i.e., only $65 \mathrm{nS}$ at room temperature. The intrinsic buckle conductance appears to be about $1 \mu \mathrm{S}$ at room temperature. This is much lower than the fourterminal conductance value of order $100 \mu \mathrm{S}$ that we typically find for nonmanipulated straight nanotubes in a similar layout. The effect of the buckle on the electron transport is thus quite dramatic. The buckle conductance is also much lower than the quantum conductance unit of $4 e^{2} / h$
$=154 \mu \mathrm{S}$, which indicates that the buckle acts as a tunnel barrier.

In Fig. 2 the conductance $G$ of the buckled segment is plotted versus absolute temperature $T$ on a doublelogarithmic scale for both the two- and four-terminal configuration. At high temperatures the data can be fitted with a power-law function $G \propto T^{\alpha}$ (solid lines). Below $120 \mathrm{~K}$, Coulomb blockade sets in which further suppresses the conductance. The power-law exponent $\alpha$ is found to be very different, $\alpha=0.26$ versus 1.4 , for the two- and four-terminal measurements respectively. The intrinsic buckle conductance (four-terminal data) thus appears to be much more strongly temperature dependent than the contact conductance (twoterminal data).

We can understand these findings on the basis of a Luttinger liquid model. The Luttinger model ${ }^{14,15}$ has been employed to explain recent transport experiments on metallic carbon nanotubes. ${ }^{16,5}$ In this model, electron-electron correlations combined with the one-dimensional nature of nanotubes lead to a power-law suppression of the tunneling conductance as a function of energy, $d I / d V \propto E^{\alpha}$. Here $E$ is the maximum of the thermal or voltage energy scale, i.e., $k_{B} T$ or $e V$ respectively, with $k_{B}$ Boltzmann's constant and $e$ the electron charge. At low bias voltages $V \ll k_{B} T / e$ this leads to a power-law behavior of the conductance as a function of $T$, i.e., $G \propto T^{\alpha}$. At high voltages $V \gg k_{B} T / e$, however, it yields a power-law dependence on voltage, $d I / d V \propto V^{\alpha}$. The exponent $\alpha$ depends on the strength of the electron-electron interactions which is characterized by the Luttinger interaction parameter $g .{ }^{14-16}$ For repulsive interactions, $g$ ranges from 0 (very strong interactions) to 1 (no interactions). Estimates of $g$ for carbon nanotubes are in the range of $0.2-0.3{ }^{14-16}$ The exponent $\alpha$ also depends on the position of tunneling. When electrons are added to the end of the nanotube, the excess electron charge can spread away in one direction only and the tunnel conductance is suppressed strongly with an exponent $\alpha_{\text {end }}=(1 / g-1) / 4$. Tunneling into the bulk of the nanotube is more weakly suppressed, with $\alpha_{\text {bulk }}=(1 / g+g$ $-2) / 8$, because the excess charge can now spread in both directions away from the contact.

The conductance of the buckle is suppressed with a power-law exponent $\alpha=1.4$ (Fig. 2). If the buckle acts as a tunnel barrier, transport across the buckle takes place by tunneling of electrons from the end of one nanotube segment to the end of the other segment. This end-to-end tunneling is associated with an exponent twice as large as tunneling into a single end, i.e., $\alpha_{\text {end }- \text { end }}=2 \alpha_{\text {end }}=(1 / g-1) / 2$. Solving $\alpha_{\text {end }- \text { end }}=1.4$ yields a Luttinger interaction parameter value $g=0.26$. In the two-terminal configuration, however, the contacts limit the conductance and one thus probes bulk tunneling from the contacts to the nanotube. Here we find $\alpha_{\text {bulk }}=0.26$, from which we obtain the same Luttinger parameter value $g=0.26$. It is gratifying that these exponents which are differing by a factor 6 , can be reconciled by this single parameter $g$. The value of $g=0.26$ is also well in agreement with theoretical estimates, ${ }^{14,15}$ recent experiments in a different geometry, ${ }^{5}$ and the value of $g=0.29 \pm 0.04$ that we find for many samples with straight nonmanipulated nanotubes. We thus conclude that the transport characteristics of this buckle are well described by assuming that it acts 

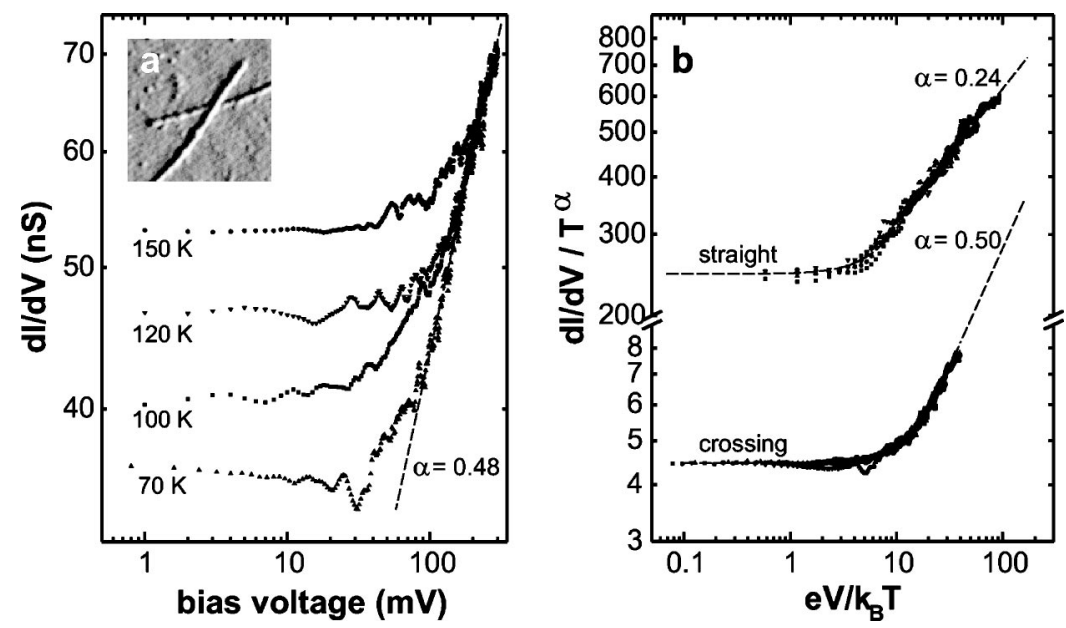

FIG. 3. Differential conductance of a manipulated nanotube crossing as a function of applied bias voltage for several temperatures. For this sample, the Coulomb blockade effect suppresses the conductance below $70 \mathrm{~K}$. At low bias voltages, $d I / d V(V)$ is constant while it depends as a power-law on temperature. At high voltages, the differential conductance crosses over to a power-law dependence on bias voltage $d I / d V \propto V^{\alpha}$, with $\alpha=0.48$ (dashed line). The inset of (a) shows a $200 \times 200 \mathrm{~nm}^{2}$ AFM amplitude image of the crossing. (b) presents a scaling plot, where $d I / d V$ has been scaled by $T^{\alpha}$ and is plotted versus $e V / k_{B} T$ for the crossing segment and, for comparison, for a typical straight segment of a nanotube.

as an artificially created nanometer-size tunnel junction within an individual nanotube.

We now discuss data for the nanotube-crossing sample shown in Fig. 1(d). The conductance of the crossing reads 80 $\mathrm{nS}$ at room temperature. ${ }^{17}$ Again this value is much lower than the conductance quantum indicating that the crossing also acts as a tunnel junction. The conductance again decreases as a power-law upon lowering the temperature, with $\alpha=0.50$ (not shown). For this sample, the Coulomb blockade effect further suppresses the conductance below $70 \mathrm{~K}$. The bias dependence of the differential conductance at several temperatures is shown in Fig. 3. At all temperatures, the data show the same behavior: At low applied bias, $d I / d V$ is constant at a level that scales as a power law with temperature $(\alpha=0.50)$. At high bias voltage it crosses over to a power-law voltage dependence, i.e., $d I / d V \propto V^{\alpha}$ with $\alpha=0.48$ (dashed line). The dependence of the differential conductance on both energy scales $e V$ and $k_{B} T$ is emphasized in Fig. 3(b), where the differential conductance is scaled by $T^{\alpha}$ and plotted versus $e V / k_{B} T$. As expected, all the data obtained at different temperatures and bias voltages collapse onto a single curve, which is well described by the theoretically expected form (dashed line). ${ }^{18}$ The exponent $\alpha$ that has been used to scale these curves onto each other is 0.50. Transport between crossing nanotubes was studied recently, but only in the low-bias regime, where this powerlaw behavior was not observed. ${ }^{10}$

The crossing junction thus yields a significantly different value $\alpha \approx 0.50$ than the buckle junction discussed above. This can be understood as a direct consequence of the particular crossing geometry. Unlike the case for the nanotube buckle where the two tube ends meet, the contact in the crossing is now from the bulk of one tube to the bulk of the other. The electron transport thus takes place via bulk-tobulk tunneling ${ }^{19}$ with an exponent that is twice as large as that for regular bulk tunneling, i.e., $\alpha_{b u l k-b u l k}=2 \alpha_{\text {bulk }}$ $=(1 / g+g-2) / 4$. From $\alpha_{b u l k-b u l k}=0.50$ we find $g=0.27$, which again is in excellent agreement with the other results for $g$. The bulk-to-bulk tunneling observed for the crossing can be readily compared to the regular bulk-tunneling configuration, which is done in Fig. 3(b), where the scaled differential conductance is shown for a straight nanotube as well. ${ }^{18}$ In this case, the exponent is found to be $\alpha=0.24$, which indeed is half the exponent observed for bulk-to-bulk tunneling. Molecular dynamics simulations have suggested that crossing nanotubes can be both deformed by about $20 \%$ at the crossing point due to the van der Waals binding of the upper nanotube to the substrate away from the crossing. ${ }^{20}$ Apparently, this deformation, if present at all, does not electronically break up the nanotubes, since our data indicate that intertube transport occurs via bulk-to-bulk rather than through end-to-end or end-to-bulk tunneling.

Recently, transport experiments were conducted on metalmetal nanotube kink junctions formed by a pentagonheptagon defect pair located at the kink ${ }^{5}$ and naturally occurring crossing junctions. ${ }^{9,10}$ Whereas such junctions are rare objects, the present work shows that one can use an AFM to precisely define local junctions at arbitrary positions along a nanotube. The transport characteristics demonstrate that these local junctions significantly alter the electronic transport properties of carbon nanotubes. A unifying description of single nanotubes, kinks, buckles, and crossings can be obtained from the Luttinger liquid model. The manipulation technique shown here allows the fabrication of various interesting new nanotube structures. For instance, double-buckle structures can be envisioned which define a roomtemperature single-electron transistor. ${ }^{21,22}$ More generally, we expect that electromechanical effects may find their use in future nanoelectronic devices.

We thank A. van den Enden for experimental assistance and L. Balents for useful discussions. The nanotube material was kindly supplied by R.E. Smalley and co-workers at Rice University. This research was financially supported by the Dutch Foundation for Fundamental Research on Matter (FOM) and the European Community SATURN Project. 
${ }^{1}$ C. Dekker, Phys. Today 52, 22 (1999).

${ }^{2}$ S. J. Tans, A. R. M. Verschueren, and C. Dekker, Nature (London) 393, 49 (1998).

${ }^{3}$ M. Bockrath et al., Science 275, 1922 (1997).

${ }^{4}$ S. J. Tans et al., Nature (London) 386, 474 (1997).

${ }^{5}$ Z. Yao, H. W. Ch. Postma, L. Balents, and C. Dekker, Nature (London) 402, 273 (1999).

${ }^{6}$ A. Rochefort, D. R. Salahub, and Ph. Avouris, Chem. Phys. Lett. 297, 45 (1998)

${ }^{7}$ C. L. Kane and E. J. Mele, Phys. Rev. Lett. 78, 1932 (1997).

${ }^{8}$ M. B. Nardelli and J. Bernholc, Phys. Rev. B 60, R16 338 (1999).

${ }^{9}$ J. Lefebvre et al., Appl. Phys. Lett. 75, 3014 (1999).

${ }^{10}$ M. S. Fuhrer et al., Science 288, 494 (2000).

${ }^{11}$ T. W. Tombler et al., Nature (London) 405, 769 (2000).

${ }^{12}$ H. W. Ch. Postma, A. Sellmeijer, and C. Dekker, Adv. Mater. 12, 1299 (2000).

${ }^{13}$ S. Iijima, C. Brabec, A. Maiti, and J. Bernholc, J. Chem. Phys. 104, 2089 (1996).

${ }^{14}$ C. Kane, L. Balents, and M. P. A. Fisher, Phys. Rev. Lett. 79, 5086 (1997).

${ }^{15}$ R. Egger and A. O. Gogolin, Phys. Rev. Lett. 79, 5082 (1997).

${ }^{16}$ M. Bockrath et al., Nature (London) 397, 598 (1999).

${ }^{17}$ Initially the crossing nanotube structure was contacted by four electrodes, with the crossing located between the middle two electrodes. The conductance of each of the two middle electrodes were determined to be about $250 \mathrm{nS}$ at room temperature. After the initial measurements, however, the outer contacts were lost, and two-terminal measurements are reported hereafter.
${ }^{18}$ The scaling functions read

$$
\begin{aligned}
(d I / d V) / T^{\alpha} \propto & \sinh (\eta x / 2)|\Gamma(1+\alpha / 2+i \eta x / 2 \pi)|^{2} \\
& \times\left\{\frac{1}{2} \operatorname{coth}(\eta x / 2)-1 / \pi \operatorname{Im}[\Psi(1+\alpha / 2+i \eta x / 2 \pi)]\right\}
\end{aligned}
$$

for the bulk-to-bulk tunneling expected for the crossing, and $(d I / d V) / T^{\alpha} \propto \cosh (\gamma x / 2)|\Gamma[(1+\alpha) / 2+i \gamma x / 2 \pi]|^{2}$ for a straight tube. Here, $x \equiv e V / k_{B} T, \gamma$ depends on the ratio of the contact conductances, $\eta$ accounts similarly for the fraction of the applied bias voltage that drops at the contacts to the nanotube, and $\Gamma$ and $\Psi$ are the gamma and digamma functions, respectively. Using $\gamma$ and $\eta$ as fitting parameters, we find $\gamma=0.6$ and $\eta=0.18$.

${ }^{19} \mathrm{~A}$ contact qualifies as a bulk contact if the distance from the contact to the end of the nanotube $L>h v_{F} / 2 \pi k_{B} T$, where $v_{F}$ is the Fermi-velocity and $h$ is Planck's constant (Ref. 23). In the sample presented in Fig. 1(d), the ends are separated from the crossing point by 110 and $130 \mathrm{~nm}$, which means that all measurements performed at temperatures above $56 \mathrm{~K}$ will show bulk-to-bulk tunneling.

${ }^{20}$ T. Hertel, R. E. Walkup, and Ph. Avouris, Phys. Rev. B 58, 13870 (1998).

${ }^{21}$ L. Chico, M. P. López Sancho, and M. C. Munoz, Phys. Rev. Lett. 81, 1278 (1998).

${ }^{22}$ M. S. C. Mazzoni and H. Chacham, Phys. Rev. B 61, 7312 (2000).

${ }^{23}$ L. Balents, cond-mat/9906032 (unpublished). XVIII Moriond Les Arcs Conference Proceedings, edited by D. C. Glattli and M. Sanquer (Edition Frontiers, Paris, France, 1999). 